\title{
Development of Archaeological Fieldwork Investigation System based on Smartphone
}

\author{
Xiangbin Guo ${ }^{a}$ and Zhaoxin Geng ${ }^{\text {b, * }}$ \\ School of Information Engineering, Minzu University of China, Beijing 100081, China \\ achampagne_guo@163.com, bongsir@163.com
}

Keywords: Archaeological questionnaire; Android; Smartphone; software; statistics.

\begin{abstract}
With the advent of smart phone age, it not only brings new opportunities for archaeological informatization in the fieldwork, but also gradually changes the way of exchanging and recording archaeological information. To meet the requirements of the archaeological application, the archaeological questionnaire system based on Android 4.4 smart phone has been developed. It could enable archaeologists to real-time deploy three different types of questionnaires, which are mainly associated with archaeological hot spots, museums and common sense. All results of the archaeological questionnaire could be calculated, stored and analyzed by using this smart phone software. Meanwhile, the latest archaeological reports could be collected and uploaded through the internet. This archaeological questionnaire system would overthrow traditional archaeological investigation in the fieldwork and provide the convenience for the archaeologists.
\end{abstract}

\section{Introduction}

With the development of the information technology, Chinese archaeologists gradually have applied intelligentized technique and portable device to the sphere of archaeology and the preservation of cultural relics [1]. Particularly, in recent years, various promoting techniques wildly utilized in archaeology are booming, such as cloud computing, big data, virtualization and mobile internet, which indeed accelerate the process of archaeological informatization [2].

However, during the research phase of an archaeological excavation, the archaeologists usually have to exploit bunches of paper questionnaires to acquire related information from some interviewees. Obviously, this inefficient behavior exerts many negative effects. For one thing, because of the use of massive papers, it not only rapidly consumes national resources, but also seriously damages our environment. For another, when archaeologists start to investigate, they have to issue multiple paper questionnaires, which is time and energy consuming. Also, analyzing the statistical data of the questionnaires also intensifies their burden to complete every investigation. Therefore, an alternative scheme is in urgent need to solve these problems. Namely, the archaeology realm calls for an archaeological fieldwork investigation system based on Android Smartphone.

It is reported that there were approximately 7 billion cellphone subscribers in the world by the end of 2013, and this enormous volume makes the cellphone hardware and software extremely cost-effective and yet rather powerful and reliable [3]. In addition, as of 2013, Android devices sell more than Windows, iPhone operating system (OS), and Mac OS X devices combined [4]. Considering the excellent characteristics of cellphone and the popularity of the Android system, we offer a solution to solve those problems caused by the traditional archaeological fieldwork. The archaeological fieldwork investigation system operating on smartphone enables archaeologists to refresh the content of questionnaires, collect the statistics of the interviewees and acquire latest archaeological information from the internet. The design and realization of this software are detailed from the three functions mentioned above. Meanwhile, a series of advices and prospects of this system are also put forward. 


\section{Software Platform}

This questionnaire application runs on the Android (ver4.4) system. What is more, to store the content and statistics of the questionnaires, this application should call SQLite database. The detailed data about this archaeological fieldwork investigation system is described as follows.

Android System. The Android system adopts hierarchical architecture as well as other operating systems. This system divides into four layers, which are Linux kernel, run-time libraries, application framework and applications, respectively, from the bottom to the top [5]. This application belongs to the top layer of Android. In other words, the application possesses not only humanized communication interface, but also the safe and reliable platform. Before developing any specific program, we must figure out the operating mechanism and the basic components of the Android system. As for the operating system, when any application firstly runs on the Android system, it will create a completely new Virtual Machine, which provides an exclusive execution environment [6]. Meanwhile, Android system consists of four building blocks: Activity, Service, Broadcast Receiver and Content Provider, which are essential to Android developers to make perfect programs [7].

SQLite Database. SQLite is a software library that implements a self-contained, server-less, zero-configuration, transactional SQL database engine [8]. And it is the most widely deployed SQL database engine in the world. We are free to access the source code of SQLite. In this paper, according to actual demands of the archaeological survey, we design some suitable database tables in SQLite to save the content of different questionnaires and the statistics of interviewees' choices. There is a main database table, which is shown as Table 1, to illustrate how the SQLite works.

Table 1 The database table of questionnaire

\begin{tabular}{|c|c|c|c|}
\hline Field Name & Data Type & Constraints & Description \\
\hline id & integer & Required & Key(Unique) \\
\hline TypeQues & varchar & Required & Type of Questionnaire \\
\hline Title & varchar & Required & Title of Questionnaire \\
\hline Time & varchar & Required & Time of Creating Questionnaire \\
\hline Question & varchar & Required & Questions \\
\hline Answer_A & varchar & Optional & Answer A \\
\hline Answer_B & varchar & Optional & Answer C \\
\hline Answer_C & varchar & Optional & Answer D \\
\hline Answer_D & varchar & Optional & Number of Choosing A \\
\hline Answer_A_Num & int & Optional & Number of Choosing B \\
\hline Answer_B_Num & int & Optional & Number of Choosing C \\
\hline Answer_C_Num & int & Optional & Number of Choosing D \\
\hline Answer_D_Num & int & Optional &
\end{tabular}

\section{Design of Archaeological Application}

Before starting to develop the archaeological fieldwork investigation system, first, the information of the archaeology field through discussing and acquiring the archaeologists who have a lot of experiences in archaeological fieldwork investigation. Combing the need of the archaeological fieldwork investigation, mobile intelligentized device and information technology, the detailed requirements document of this Android application was drawn up. It is mainly concerned with how to satisfy archaeologists and interviewees. Therefore, this application mainly could offer three typical functions, which is shown in Fig.1. 


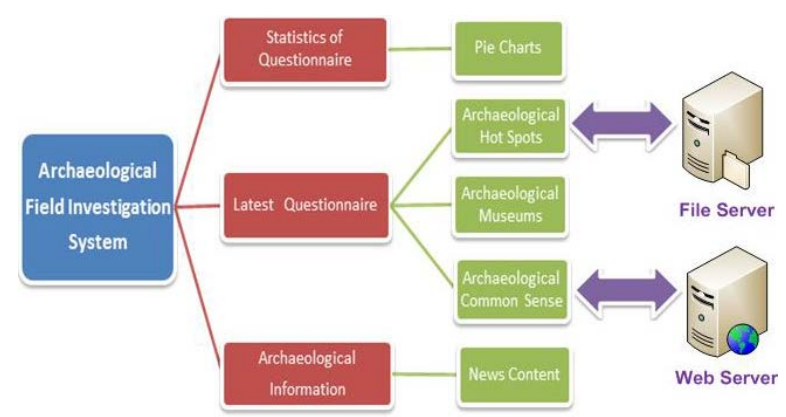

Fig. 1 The three function modules of the application

The most important function of the archaeological fieldwork investigation system is that it enables archaeologists to upload and refresh different questionnaires whenever they need wherever they are. Meanwhile, the questionnaires are also divided into three categories, which are archaeological hot spots, museums and common sense. For the first part, archaeological hot spots, its main purpose is to follow the hot archaeological news and keep this software updated any time. As the second part, archaeological museum, the reasons why its function was added are not only to suggest publics take more care of historical relics, but also to improve the standard of museums service. However, what we could make significant progress on archaeological museums is recorded in public's feedback. The last one, archaeological common sense, it primarily focuses on promoting the popularity of human archaeological knowledge.

When interviewees finish their questionnaires on the Smartphone every time, archaeologists could immediately check how many members participate in this survey and obtain specific percentages of different answers from the pie charts, which indeed release some of their burden. At last, to timely acquire archaeological information from the internet, archaeologists only need to click the refresh button. This archaeological questionnaire application is really able to simplify and facilitate archaeologists' work and life to a large extent.

\section{Results and Discussion}

To expose the three main functions of this application to customers directly, we decide to implement them by applying FragmentActivity which extends from Activity. Meanwhile, three fragments are added in this FragmentAcitity to load three aspects of content, which are archaeological questionnaires, statistics and information. When customers prefer some part of the content, they just need to click the RadioButton at the bottom of the screen, which can switch to the corresponding page.
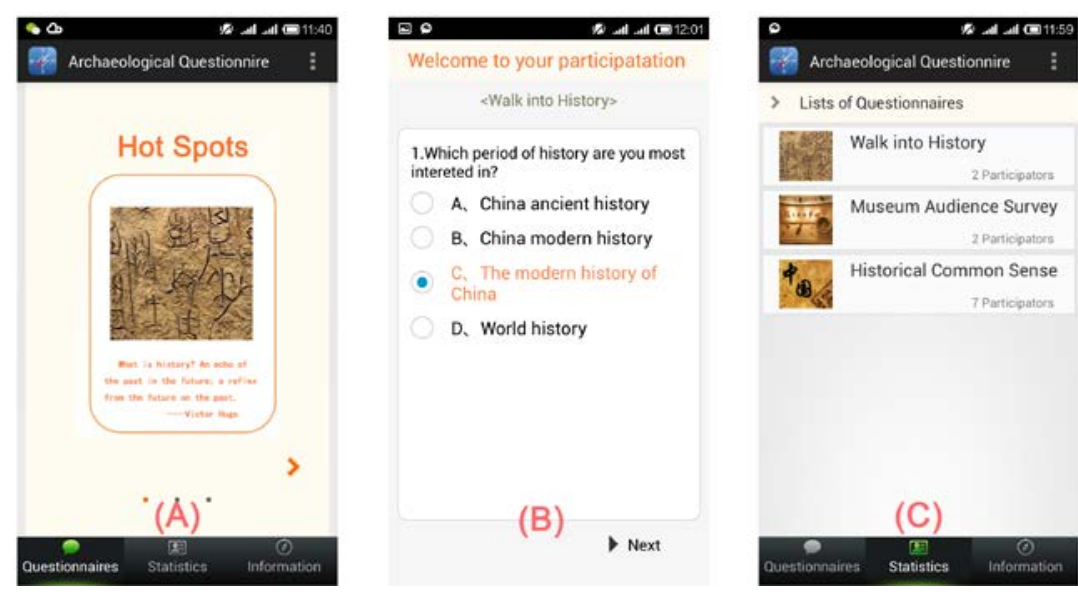

Fig.2 (A) The archaeological hot spots, (B) List of questionnaires, (C) Participate in an investigation

Once interviewees launch this application on their Smartphone, the archaeological questionnaire page, the default one (as shown in Fig.2 (A)), would show three options to them, which are archaeological hot spots, museums and common sense. What they need to do is to slide three pages and click them. To implement this novel function, ViewPager is called by our developers, who supply an implementation of a PagerAdapter to generate the pages, to satisfy the users. When the users take 
part in responding the questionnaires, they are free to choose one answer and then click the Next button (as shown in Fig.2 (B)). After they finish the questionnaires once, this program will automatically list the process of all questionnaires (as shown in Fig.2 (C)).

Pie Chat and Loading Data. Although one main purpose of this application is to completely store questionnaires statistics, we need a better way to visually display the data. Therefore, our developers decide to import others' jar package into the program, which can easily show the specific percentages of different answers in the pie chart (as shown in Fig.3 (A)). Furthermore, when the archaeologist clicks one part of the colorful pie, it will pop up the corresponding data friendly. The above functions mainly fulfill the archaeologists' requirements about questionnaires. Moreover, this application can also offer them access to loading the latest archaeological information. It mainly utilizes Service component to create a thread to asynchronously acquire the relevant data from the Internet, and displays them in a List View on the screen (as shown in Fig.3 (B)). If the archaeologist is interested in some news, they just need to click the corresponding items. The news will be exposed to them in detail.

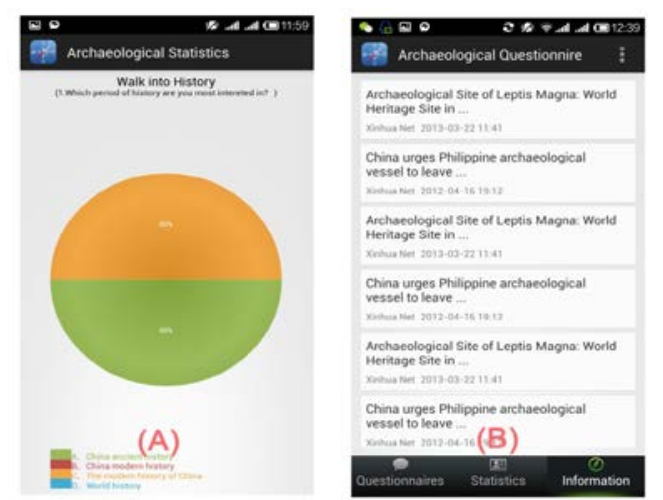

Fig.3 (A) Statistics of questionnaire, (B) The latest archaeological information

\section{Conclusion and Prospects}

Once this archaeological fieldwork investigation system has been installed on the Smartphone, it can immediately provide better service to archaeologists. Recently, some archaeological teams have already applied our product to their projects, and they offer us some positive comments. Hence, we start to be more confident with our product. Nevertheless, if some flaws can be modified properly, it is deemed to be promising. Therefore, the urgent task is to enrich the content of the application and optimize man-machine interface, which contributes to improving the user experience. Meanwhile, creating a cloud platform to share archaeological information should be taken into consideration in the future, because these collected data can be utilized to analyze and predict the behavior of archaeologists and interviewees. We believe that this archaeological fieldwork investigation system will step forward for the development of intelligentialization and simplification.

\section{Acknowledgment}

The project was supported by Science and Technology Research Funding of State Cultural Relics Bureau (No.20110135), “985” Project and The Independent Scientific Research of MUC (10301-01402904).

\section{References}

[1] Jianhua Liu. The application of computer technology in archaeology and conservation of cultural relics. Cultural Relics of Central China. 5, 75-80 (2004).

[2] Chunye Ni. Discussion on the influence of network technology to the future of archaeological development. Heilongjiang Chronicles. 11, 203-204 (2010). 
[3] O. Aydogan. Mobile phones democratize and cultivate next-generation imaging, diagnostics and measurement tools. Royal Society of Chemistry. 14(17), 3187-94 (2014).

[4] Android's Google Play beats App Store with over 1 billion apps, now officially largest. Phonearena.com, (2013).

[5] Hongjun Ni and Changjun Qian: Design and implementation of spontaneous SMS based on the Android platform. Application of Electronic Technique. 38(12), 126-129 (2012).

[6] Zhihai Yang, Weihong Yu, Yan Chen and Taoying Li: Mobile Healthcare Research Based on Jade Agent and Android Platform. International Conference on Software Engineering and Service Science. IEEE, 166-N111 (2014).

[7] Android on http://www.android.com.

[8] SQLite on http://www.sqlite.org/. 